\title{
Research Paper: The Effect of Repetitive Transcranial Mag- netic Stimulation on Postural Stability After Acute Stroke: A Clinical Trial
}

Bijan Forogh ${ }^{1}$, Tannaz Ahadi ${ }^{1}$, Maryam Nazari ${ }^{1}$, Simin Sajadi ${ }^{1}$, Lydia Abdul Latif ${ }^{2}$, Seyed Majid Akhavan Hejazi $^{2}$, Gholamreza Raissi ${ }^{*}$

1. Neuromusculoskeletal Research Center, Firoozgar Hospital, Tehran, Iran.

2. Department of Rehabilitation Medicine, Faculty of Medicine, University of Malaya, Kuala Lumpur, Malaysia.

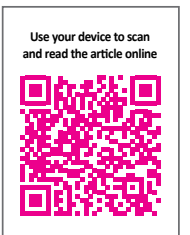

Citation: Forogh, B., Ahadi, T., Nazari, M., Sajadi, S., Abdul Latif, L., Akhavan Hejazi, S. M., et al. (2017). The Effect of Repetitive Transcranial Magnetic Stimulation on Postural Stability After Acute Stroke: A Clinical Trial. Basic and Clinical Neuroscience, 8(5):405-412. https://doi.org/10.18869/nirp.bcn.8.5.405

Article info:

Received: 12 Jan. 2017

First Revision: 26 Jan. 2017

Accepted: 17 Jun. 2017
Key Words:

Stroke, Transcranial magnetic stimulation, Stroke rehabilitation, Postural balance, Proprioception

\begin{abstract}
A B S T RA C T
Introduction: Balance impairment is a common problem and a major cause of motor disability after stroke. Therefore, this study aimed to investigate whether low-frequency repetitive Transcranial Magnetic Stimulation (rTMS) improves the postural balance problems in stroke patients.

Methods: This randomized double blind clinical trial with 12 weeks follow-up was conducted on stroke patients. Treatment was carried with $1 \mathrm{~Hz} r T M S$ in contralateral brain hemisphere over the primary motor area for 20 minutes (1200 pulses) for 5 consecutive days. Static postural stability, Medical Research Council (MRC), Berg Balance Scale (BBS), and Fugl-Meyer assessments were evaluated immediately, 3 weeks and 12 weeks after intervention.

Results: A total of 26 patients were enrolled (age range $=53$ to 79 years; $61.5 \%$ were male) in this study. Administering rTMS produced a significant recovery based on $\mathrm{BBS}(\mathrm{df}=86,7 ; \mathrm{F}=7.4$; $\mathrm{P}=0.01)$, Fugl-Meyer Scale $(\mathrm{df}=86,7 ; \mathrm{F}=8.7 ; \mathrm{P}<0.001)$, MRC score $(\mathrm{df}=87,7 ; \mathrm{F}=2.9 ; \mathrm{P}=0.01)$, and static postural stability $(\mathrm{df}=87,7 ; \mathrm{F}=9.8 ; \mathrm{P}<0.001)$ during the 12 weeks follow-up.

Conclusion: According to the findings, rTMS as an adjuvant therapy may improve the static postural stability, falling risk, coordination, motor recovery, and muscle strength in patients with stroke.
\end{abstract}

\section{Introduction}

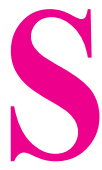

troke as the most disabling neurologic injury and third leading cause of death, is one of the most important challenges of health systems. It significantly reduces the patients' quality of life and can also increase the health care costs. The annual incidence of this disease is very high. According to the World Health Organization, 37 million cases of stroke were estimated (Mathers, Fat, \& Boerma, 2008) in 2004. Although the incidence of stroke decreased in developed countries since the early 1970s, this trend was reversed in developing countries (Feigin, Lawes, Bennett, Barker-Collo, \& Parag, 2009). Balance and postural stability impairment is a common problem and a major cause of motor disability (locomotion) after

* Corresponding Author:

Gholamreza Raissi, $M D$

Address: Neuromusculoskeletal Research Center, Firoozgar Hospital, Tehran, Iran.

Tel:+98 (21) 82141612

E-mail: grezaraissi@yahoo.com 
stroke (Lee, 1989; Rode, Tiliket, \& Boisson, 1997). Today, several tools and techniques are used to improve poststroke postural balance and performance. Among them, repetitive transcranial magnetic stimulation (rTMS) has been used as an adjuvant therapy (Dimyan \& Cohen, 2010).

The first successful rTMS study was performed in 1985 by Anthony Barker and his colleagues in England (Corthout, Barker, \& Cowey, 2001). It is a simple and non-invasive procedure that can have positive effects on motor recovery in post-stroke hemiparesis (Dimyan \& Cohen, 2010; Weiduschat et al., 2011). This procedure has been used in two methods: low-frequency stimulation $(\leq 1 \mathrm{~Hz})$ to decrease the excitability of the unaffected brain hemisphere or high-frequency stimulation $(>1 \mathrm{~Hz})$ to increase excitability of the affected brain hemisphere (Forogh, Yazdi-Bahri, Ahadi, Fereshtehnejad, \& Raissi, 2014; Hao, Wang, Zeng, \& Liu, 2013; Khedr, Abdel-Fadeil, Farghali, \& Qaid, 2009; Khedr, Etraby, Hemeda, Nasef, \& Razek, 2010). The effect of rTMS on balance is still unclear. Since rTMS may improve patient's motor recovery, it may have a positive effect on functional balance in the subacute and chronic phase of stroke. Therefore, this study aimed to investigate whether low-frequency rTMS improves the balance problems in stroke patients.

\section{Methods}

\subsection{Study design and setting}

This randomized double blind clinical trial with 12 weeks follow-up was conducted in Firoozgar hospital, Tehran, Iran between April to December 2014. Patients were selected con-secutively from those who were admitted at Firoozgar Center of Physical Medicine and Rehabilitation. The protocol of the study was approved by the Ethics Committee of Iran Univer-sity of Medical Sciences. Signed informed consent was attained from all patients prior to enrollment in the study.

\subsection{Subjects}

Inclusion criteria were as follows: stroke patient with subacute and chronic ischemic and hemiplegic stroke documented by Computed Tomography (CT) or MRI; at least one month has elapsed from stroke; first-ever cerebral infarction; ability to perform 3-step command (3 points); no cognitive impairment, impaired patient's balance and gait; the ability to walk with or without support; and with Functional Ambulation Categories more than one.

Patients were not included in the study if they had: a second stroke, bilateral weakness; the cerebel-lum or brain stem involvement, proprioception impairment hemianopsia or another visual impairment, vestibular dysfunction, neurologic comorbidity other than stroke like neuropathy, severe postural instability, orthopedic problems, significant cognitive problem, receptive aphasia, epilepsy or seizures after stroke, and pathological conditions referred as contraindica-tions of rTMS (presence of a metallic implant inside the eye or the brain, the external fixator, cardiac pacemaker). For sample size calculation, according to Emara et al., (2010) study, with considering the type I error equal to $5 \%(\alpha=0.05)$ and an accuracy of $1 \%(\mathrm{~d}=0.01)$, the number of patients required in each group was found as 10. Randomization was done by an independent researcher. Subjects were randomly assigned to two groups; rTMS and Sham group.

\subsection{Intervention}

Treatment was carried in 5 consecutive days, with $1 \mathrm{~Hz}$ rTMS in contralateral brain hemisphere over the primary motor area for 20 minutes (1200 pulses), in sitting position. Low-frequency rTMS was administered by a 70 mm figure-8 coil connected to Magstim R30 stimulator (MagVenture, Denmark). The optimal site and intensity of stimulation was deter-mined based on proposed method of Kondo et al., (2013). As in the real rTMS group, for Sham stimulation, we recorded the sound of stimulator. A small speaker was installed on the stimulation coil handle. The coil was placed on the head, adjustments were done on the rTMS monitor, but speaker was activated by a switch behind the patient. A sound mimicking the real rTMS was played for the patient (Figure 1).

\subsection{Measurement}

Clinical and postural evaluations were performed prior to the first session, immediately after the rTMS course, 3 weeks and then 3 months later. Static postural stability was assessed by a balance assessment system (Biodex, Balance System SD, 115 VAC, Germany). For static postural stability, the patients' standing on a stable flat were evaluated. The patient's legs were placed on 30-degree angle for 20 to 60 seconds and they were asked to maintain their standing balance. Balance function without external perturbation and the open as well as closed eyes was evaluated. Medical Research Council (MRC) scale was used to assess muscle strength (Paternostro-Sluga et al., 2008). This scale is a six grade scoring system in which 0 denotes no movement and 5 normal power. Static and dynamic balance ability was evaluated by Berg Balance Scale (BBS) (Steffen, Hacker, \& Mollinger, 2002). It comprises 14 items and each item is scored from 0 (unable) to 4 (independent) with maximum total 


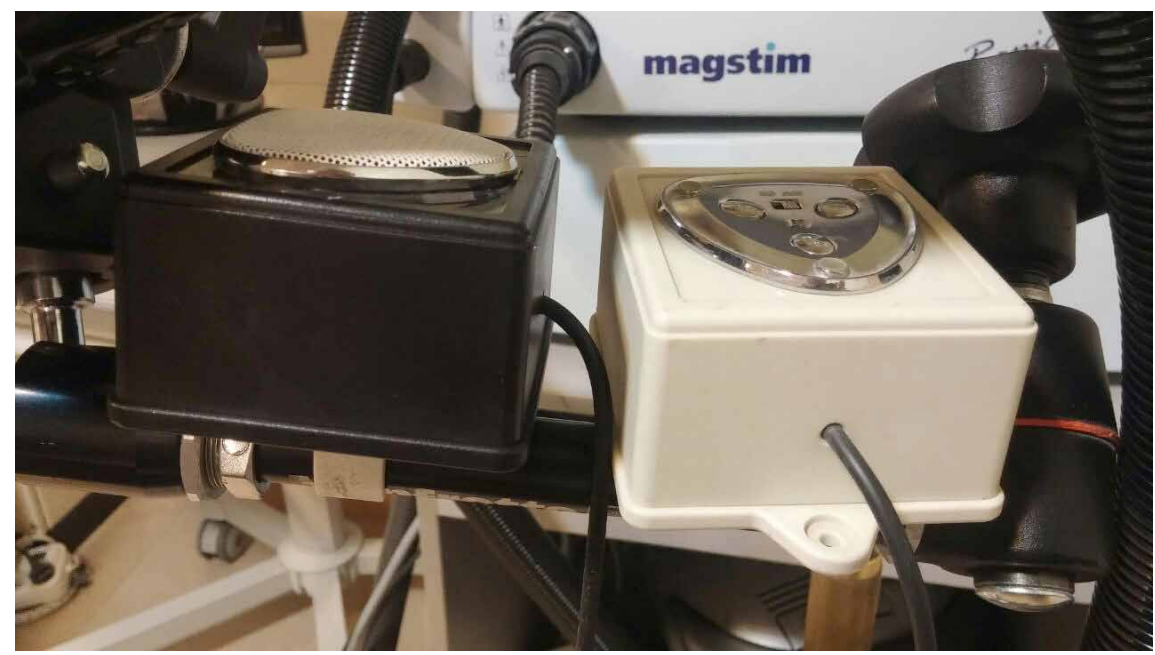

Figure 1. The device was installed for Sham stimulation.

NEUR:SCIENCE

score of 56. Finally, Fugl-Meyer assessment was used to assess motor recovery after stroke. It is scored on a 3 -point ordinal scale (0-2) with maximum of 226 (Atler, Malcolm, \& Greife, 2015).

\subsection{Data analysis}

SPSS 22.0 was used for statistical analysis. Descriptive and inferential statistics including repeated measures ANOVA to assess trends of improvement within each group, 2-way ANOVA for detection of statistical difference of recovery between two groups over the time were used. In all analyzes, $\mathrm{P}<0.05$ was considered as significant.

\section{Results}

A total of 26 patients were enrolled (age range $=53$ to 79 years; $61.5 \%$ were male) in this study. Left brain hemisphere was affected in $18(69.2 \%)$ patients. Duration of the disease in $22(84.6 \%)$ was more than 6 months. During 12 weeks follow-up, 11 patients ( 5 patients in the treatment and 6 patients in Sham group) withdrew from the study.

Administration of rTMS produced a significant recovery in BBS during 12 weeks follow-up (compared with preintervention time). Mean(SD) BBS of rTMS group at baseline was 44.6(5.2), after 5 sessions of rTMS, 3 weeks and 12 weeks later it reached to 47.6(4.4), 49.6(4.4) and 50.1(3.9), respectively ( $\mathrm{df}=3$; $\mathrm{F}=7.5 ; \mathrm{P}=0.004)$. Compared with Sham group, BBS in patients treated with rTMS after 3 weeks [49.6(4.4) vs. 46(44); $\mathrm{P}=0.03$ ] and 12 weeks [50.1(3.9) vs. 46.7(5.8); $\mathrm{P}=0.02]$ showed a significant increase $(\mathrm{df}=7,86 ; \mathrm{F}=7.4$; $\mathrm{P}=0.01$ ) (Figure 2).
Administration of rTMS improved motor recovery after stroke during 12 weeks follow-up (compared with preintervention time). Mean(SD) Fugl-Meyer Scale at baseline in rTMS group was 22.7(6.1). The Mean(SD) score immediately, three weeks and 12 weeks after rTMS, reached to 24.3(4.9), 26.2(4.2) and 28.7(4.2), respectively $(\mathrm{df}=3 ; \mathrm{F}=15.3 ; \mathrm{P}<0.001)$. Mean(SD) Baseline Fugl-Meyer score in rTMS group was significantly lower than Sham group $(\mathrm{df}=86,7$; $\mathrm{F}=8.7 ; \mathrm{P}<0.001$ ) while 3 months after treatment it became nearly the same score $[29.0(2.6)$ vs. 28.7(4.3); $\mathrm{P}>0.99$ )] (Figure 3).

Treatment with rTMS resulted in significant increase in muscle strength (Figure 4). Although the

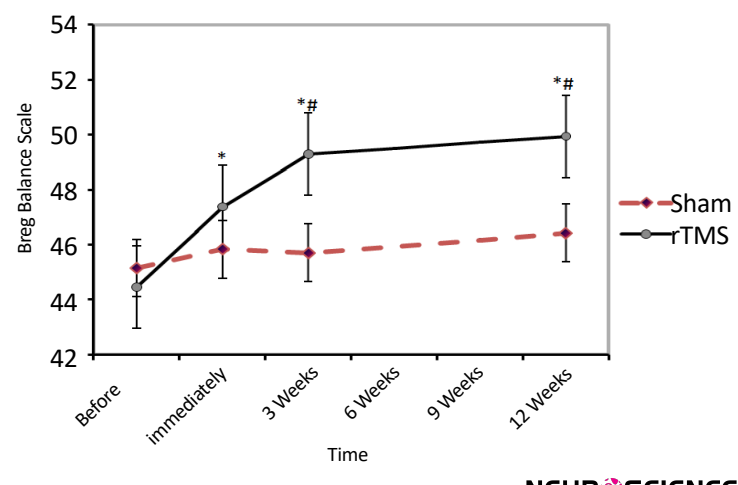

\section{NEUR SCIENCE}

Figure 2. The impact of rTMS on functional balance during 12 weeks follow-up of patients, based on the Berg Balance Scale

* Indicates a significant difference with the pre-intervention period.

\# Represents a significant difference with the corresponding time in Sham group.

No data were collected during 6- and 9-weeks periods. 


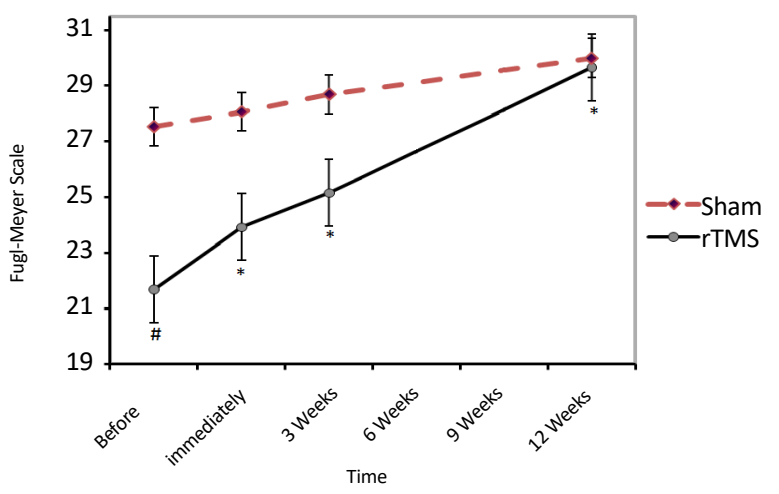

NEUR:SCIENCE

Figure 3. The impact of rTMS on motor recovery during three weeks of follow-up based on the Fugl-Meyer Scale.

* Indicates a significant difference with the pre-intervention period.

No data were collected during 6- and 9-weeks periods.

Mean(SD) MRC score in rTMS group did not change after 5 sessions [3.8(0.8) compared with 3.7(0.9); $\mathrm{df}=2 ; \mathrm{F}=1.0 ; \mathrm{P}=0.35]$, three weeks and 12 weeks after treatment it significantly increased to $4.4(0.5)$ and 4.6(0.5), respectively $(\mathrm{df}=3, \mathrm{~F}=13.8 ; \mathrm{P}<0.001)$. Twoway ANOVA revealed the Mean(SD) MRC in rTMS group compared with Sham significantly improved at the 3 weeks [4.4(0.5) vs. 3.6(0.9); $\mathrm{P}=0.03$ ] and 12 weeks $[3.9(0.8)$ vs. $4.4(0.5) ; \mathrm{P}=0.04]$ after treatment $(\mathrm{df}=7,87 ; \mathrm{F}=2.9 ; \mathrm{P}=0.01)$.

Static postural stability was improved in rTMS group over 12 weeks (Figure 5). This improvement was significant compared with before intervention $(\mathrm{df}=3 ; \mathrm{F}=7.7 ; \mathrm{P}<0.00)$ and cor-responding times in Sham ( $\mathrm{df}=7 ; 87 ; \mathrm{F}=9.8 ; \mathrm{P}<0.001)$. Mean(SD) MRS scores for the 3 weeks and 12 weeks after rTMS in treatment group were 1.12(0.6) and 1.14(0.6), respectively while in the Sham group they were 1.6(0.6) and $1.85(0.4)$, respectively.

\section{Discussion}

The findings of this study showed that rTMS as an adjuvant therapy can significantly improve the static postural stability, functional recovery and muscle strength in patients with stroke. To our knowledge, this is the first study on the role of rTMS on balance stability. However, several studies have demonstrated beneficial impact of rTMS on motor recovery after stroke. For example, Khe$\mathrm{dr}$ et al. showed the beneficial effect of rTMS on stroke related dysphagia and functional recovery (Khedr et al., 2009; Khedr et al., 2010; Khedr et al., 2014). Avenanti et al. concluded that combined time-locked rTMS was an effective and encouraging method for improvement of

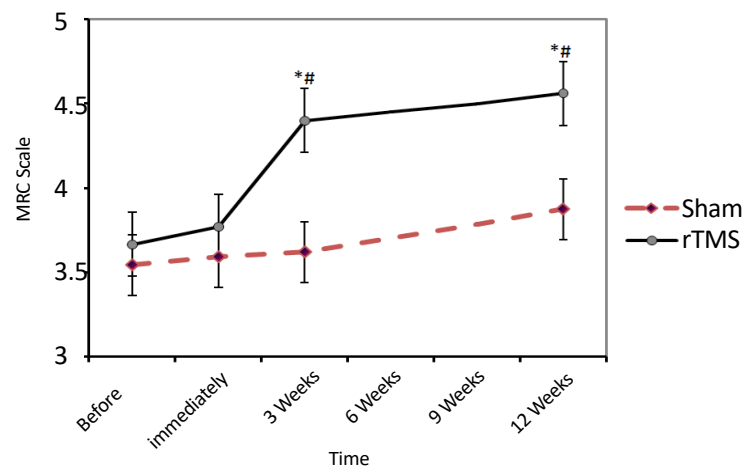

NEUR SCIENCE

Figure 4. The impact of rTMS on muscle strength during 12 weeks follow-up based on the Medical Research Council (MRC) scale.

* Indicates a significant difference with the pre-intervention time. \# Represents a significant difference with the corresponding time in the Sham group.

No data were collected during 6- and 9-weeks periods.

chronic stroke patients with mild motor impairment (Avenanti, Coccia, Ladavas, Provinciali, \& Ceravolo, 2012).

In addition, Corti et al. in their review suggested that rTMS applied to the affected brain hemisphere was a safe method and could be considered as a valid technique for restraining brain function and contributing to motor recovery after stroke (Corti, Patten, \& Triggs, 2012). Takeuchi et al. demonstrated that rTMS was a promising non-invasive tool for the hand function recovery (Takeuchi, Chuma, Matsuo, Watanabe, \& Ikoma, 2005). Emara and colleagues also showed that rTMS might improve post-stroke functional recovery. These re-searchers reported that the recovery rate in $1 \mathrm{~Hz}$ rTMS treated group is better than $5 \mathrm{~Hz}$ one (Emara et al., 2010).

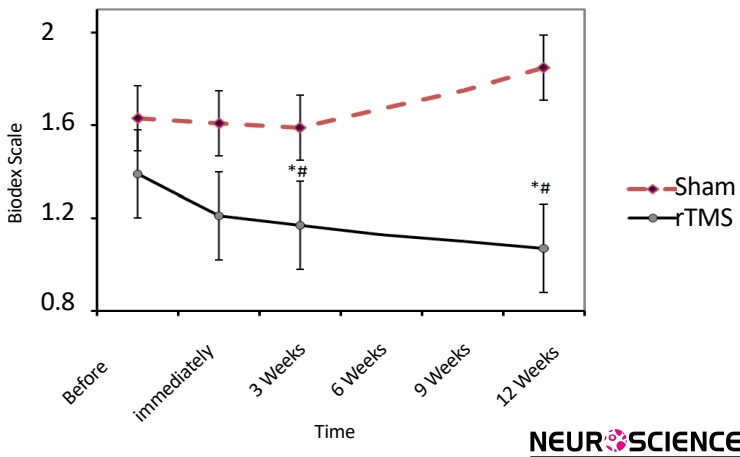

Figure 5. The impact of rTMS on the static postural stability in the 12 weeks follow-up.

* Indicates a significant difference with the pre-intervention. \#Indicating significant difference with corresponding time in Sham group.

No data were collected during 6- and 9-weeks periods. 
The underlying mechanisms of rTMS in stroke recovery have remained unclear. However, the effectiveness of these techniques in the excitability of neurons has been proved (Iyer, Schleper, \& Wassermann, 2003). rTMS use electromagnetic induction to produce an electric current across the scalp and skull without any physical contact (Eichhammer, Langguth, Marienhagen, Kleinjung, \& Hajak, 2003). Researchers generally believe that rTMS through changing the excitability of the nerve cells such as Long-Term Potentiation (LTP) and Long-Term Depression (LTD) causes an excitatory or inhibitory effect (Speer et al., 2000). Serotonin receptors, noradrenergic and dopaminergic change are also likely to be affected by rTMS (Wassermann \& Lisanby, 2001). Brain-Derived Neurotrophic Factor (BDNF) has an essential role in neuronal plasticity (Hashimoto, 2013). For example, release of BDNF after physical exercise may cause considerable modification in structure and function of astrocytes that protects against glutamate toxicity during aging and a number of neurodegen-erative disorders (Fahimi et al., 2016). Recent reports suggest that BDNF mediates, at least in part, the therapeutic effects of rTMS. Chang et al. showed that BDNF gene polymorphism has negative effect on the outcome of rTMS on the motor recovery of upper extremities in stroke patients (Chang et al., 2014).

Niimi et al. showed that the combination of rehabilitation and low-frequency rTMS may improve motor function in the affected limb, by activating brain-derived neurotrophic factor processing (Niimi et al., 2016). In the first week after the stroke, the presence of excitatory potentials in paresis limb in response to stimulation of the affected hemisphere may be a good predictor of functional recovery (Catano, Houa, Caroyer, Ducarne, \& Noel, 1995; D’Olhaberriague et al., 1997; Escudero, Sancho, Bautista, Escudero, \& López-Trigo, 1998; Hendricks, Pasman, Merx, van Limbeek, \& Zwarts, 2003; Rossini et al., 1994; Rossini et al., 1998). On the contrary, the absence of such potentials is associated with poor recovery (Shimizu et al., 2002). In addition, neuroimaging studies show that patients with poor recovery have higher levels of brain activity in unaf-fected hemisphere (Ward \& Frackowiak, 2006). This excitatory imbalance between two hemispheres, decline during the first month after stroke. This period is simultaneously associ-ated with functional improvement (Cicinelli, Traversa, \& Rossini, 1997; Delvaux et al., 2003; Traversa, Cicinelli, Pasqualetti, Filippi, \& Rossini, 1998).

The reason for using rTMS in stroke patients is based on these changes. It is believed that stroke leads to loss of inhibitory effect of damaged hemisphere on the unaffected side. When inhibition of the normal hemisphere is removed; the excitatory function of this hemisphere increases. Subsequently, inhibitory effect of normal hemisphere on affected hemi-sphere will be increased. Therefore, the use of low-frequency rTMS over the unaffected hemisphere may decrease inhibitory signals and consequently damaged hemisphere be reactivated, leading to better functional recovery. There are several studies to prove this hypothesis. For example, Mansur et al. first demonstrated that inhibition of the unaffected hemisphere by low-frequency rTMS (1 Hz) led to substantial improvement in limb performance (Mansur et al., 2005). In addition, Takeuchi et al. reported that rTMS of contralesional primary motor cortex improves hand function after stroke (Takeuchi et al., 2005).

The main limitation of this study is its low sample size that could affect the results. However, the minimum power obtained with this sample size was $81 \%$, so this limitation was largely overcome. Another limitation was short follow up period. So, we were not able to determine the long-term effects of rTMS. The present study showed that rTMS as an adjuvant thera-py may improve the static postural stability, falling risk, coordination, motor recovery, and muscle strength in patients with stroke. These effects could persist up to 3 months. Further research should be conducted with larger sample size.

\section{Acknowledgments}

This paper was part of Maryam Nazari MD thesis and supported by Iran University of Medical Sciences. The authors are thankful to Mr. Nahavandi for his technical cooperation in Sham device production and Hosniyeh Soleymanzadeh in preparation of the paper as well as doing the statistics.

\section{Conflict of Interest}

All authors certify that this manuscript has neither been published in whole nor in part nor being considered for publication elsewhere. The authors have no conflicts of interest to declare.

\section{References}

Atler, K., Malcolm, M., \& Greife, C. (2015). A follow-up study on the relationship among participation, activity and motor function in survivors of stroke following constraint-induced therapy. Disability and Rehabilitation, 37(2), 121-128. doi: 10.3109/09638288.2014.910560

Avenanti, A., Coccia, M., Ladavas, E., Provinciali, L., \& Ceravolo, M. (2012). Low-frequency rTMS promotes use-dependent 
motor plasticity in chronic stroke A randomized trial. Neurology, 78(4), 256-264. doi: 10.1212/wnl.0b013e3182436558

Catano, A., Houa, M., Caroyer, J., Ducarne, H., \& Noel, P. (1995). Magnetic transcranial stimulation in non-haemorrhagic sylvian strokes: interest of facilitation for early functional prognosis. Electroencephalography and Clinical Neurophysiology/Electromyography and Motor Control, 97(6), 349-354. doi: 10.1016/0924-980x(95)00127-7

Catano, A., Houa, M., \& Noël, P. (1997). Magnetic transcranial stimulation: Clinical interest of the silent period in acute and chronic stages of stroke. Electroencephalography and Clinical Neurophysiology/Electromyography and Motor Control, 105(4), 290-296. doi: 10.1016/s0924-980x(97)00021-0

Chang, W. H., Bang, O. Y., Shin, Y. I., Lee, A., Pascual-Leone, A., \& Kim, Y. H. (2014). BDNF polymorphism and differential rTMS effects on motor recovery of stroke patients. Brain Stimulation, 7(4), 553-58. doi: 10.1016/j.brs.2014.03.008

Cicinelli, P., Traversa, R., \& Rossini, P. (1997). Post-stroke reorganization of brain motor output to the hand: A 2-4 month follow-up with focal magnetic transcranial stimulation. Electroencephalography and Clinical Neurophysiology/Electromyography and Motor Control, 105(6), 438-50. doi: 10.1016/s0924980x(97)00052-0

Corthout, E., Barker, A., \& Cowey, A. (2001). Transcranial magnetic stimulation. Experimental Brain Research, 141(1), 128-32. doi: $10.1007 / \mathrm{s} 002210100860$

Corti, M., Patten, C., \& Triggs, W. (2012). Repetitive transcranial magnetic stimulation of motor cortex after stroke: A focused review. American Journal of Physical Medicine E Rehabilitation, 91(3), 254-70. doi: 10.1097/phm.0b013e318228bf0c

D'Olhaberriague, L., Gamissans, J. M. E., Marrugat, J., Valls, A., Ley, C. O., \& Seoane, J. L. (1997). Transcranial magnetic stimulation as a prognostic tool in stroke. Journal of the Neurological Sciences, 147(1), 73-80. doi: 10.1016/ s0022-510x(96)05312-9

Delvaux, V., Alagona, G., Gérard, P., De Pasqua, V., Pennisi, G., \& de Noordhout, A. M. (2003). Post-stroke reorganization of hand motor area: A 1-year prospective follow-up with focal transcranial magnetic stimulation. Clinical Neurophysiology, 114(7), 1217-25. doi: 10.1016/s1388-2457(03)00070-1

Dimyan, M. A., \& Cohen, L. G. (2010). Contribution of transcranial magnetic stimulation to the understanding of functional recovery mechanisms after stroke. Neurorehabilitation and Neural Repair, 24(2), 125-135. doi: 10.1177/1545968309345270

Eichhammer, P., Langguth, B., Marienhagen, J., Kleinjung, T., \& Hajak, G. (2003). Neuronavigated repetitive transcranial magnetic stimulation in patients with tinnitus: A short case series. Biological Psychiatry, 54(8), 862-65. doi: 10.1016/s00063223(02)01896-6

Emara, T., Moustafa, R., Elnahas, N., Elganzoury, A., Abdo, T., Mohamed, S., et al. (2010). Repetitive transcranial magnetic stimulation at $1 \mathrm{~Hz}$ and $5 \mathrm{~Hz}$ produces sustained improvement in motor function and disability after ischaemic stroke. European Journal of Neurology, 17(9), 1203-9. doi: 10.1111/j.14681331.2010.03000.x

Escudero, J. V., Sancho, J., Bautista, D., Escudero, M., \& LópezTrigo, J. (1998). Prognostic value of motor evoked potential obtained by transcranial magnetic brain stimulation in motor function recovery in patients with acute ischemic stroke. Stroke, 29(9), 1854-59. doi: 10.1161/01.str.29.9.1854
Fahimi, A., Baktir, M. A., Moghadam, S., Mojabi, F. S., Sumanth K., McNerney, M. W., et al. (2016). Physical exercise induces structural alterations in the hippocampal astrocytes: Exploring the role of BDNF-TrkB signaling. Brain Structure and Function, 222(4), 1797-1808. doi: 10.1007/s00429-016-1308-8

Feigin, V. L., Lawes, C. M., Bennett, D. A., Barker-Collo, S. L., \& Parag, V. (2009). Worldwide stroke incidence and early case fatality reported in 56 population-based studies: A systematic review. The Lancet Neurology, 8(4), 355-69. doi: 10.1016/s14744422(09)70025-0

Forogh, B., Yazdi-Bahri, S. M., Ahadi, T., Fereshtehnejad, S. M., \& Raissi, G. R. (2014). Comparison of two protocols of transcranial magnetic stimulation for treatment of chronic tinnitus: A randomized controlled clinical trial of burst repetitive versus high-frequency repetitive transcranial magnetic stimulation. Neurological Sciences, 35(2), 227-32. doi: 10.1007/s10072013-1487-5

Hao, Z., Wang, D., Zeng, Y., \& Liu, M. (2013). Repetitive transcranial magnetic stimulation for improving function after stroke. Sao Paulo Medical Journal, 131(6), 440-49. doi: 10.1590/15163180.20131316t2

Hashimoto, K. (2013). Sigma-1 receptor chaperone and brainderived neurotrophic factor: Emerging links between cardiovascular disease and depression. Progress in Neurobiology, 100, 15-29. doi: 10.1016/j.pneurobio.2012.09.001

Hendricks, H. T., Pasman, J. W., Merx, J. L., Van Limbeek, J., \& Zwarts, M. J. (2003). Analysis of recovery processes after stroke by means of transcranial magnetic stimulation. Journal of Clinical Neurophysiology, 20(3), 188-195. doi: 10.1097/00004691200305000-00004

Iyer, M. B., Schleper, N., \& Wassermann, E. M. (2003). Priming stimulation enhances the depressant effect of low-frequency repetitive transcranial magnetic stimulation. The Journal of Neuroscience, 23(34), 10867-72. PMID: 14645480

Khedr, E., Abdel-Fadeil, M., Farghali, A., \& Qaid, M. (2009). Role of 1 and $3 \mathrm{~Hz}$ repetitive transcranial magnetic stimulation on motor function recovery after acute ischaemic stroke. European Journal of Neurology, 16(12), 1323-30. doi: 10.1111/j.14681331.2009.02746.x

Khedr, E., Etraby, A., Hemeda, M., Nasef, A., \& Razek, A. (2010) Long-term effect of repetitive transcranial magnetic stimulation on motor function recovery after acute ischemic stroke Acta Neurologica Scandinavica, 121(1), 30-37. doi:10.1111/j.16000404.2009.01195.x

Khedr, E. M., Abo El-Fetoh, N., Ali, A. M., El-Hammady, D. H., Khalifa, H., Atta, H., et al. (2014). Dual-hemisphere repetitive transcranial magnetic stimulation for rehabilitation of poststroke aphasia. Neurorehabilitation and Neural Repair, 28(8), 740-50. doi: $10.1177 / 1545968314521009$

Kondo, T., Kakuda, W., Yamada, N., Shimizu, M., Hagino, H., \& Abo, M. (2013). Effect of low-frequency rTMS on motor neuron excitability after stroke. Acta Neurologica Scandinavica, 127(1), 26-30. doi: 10.1111/j.1600-0404.2012.01669.x

Lee, W. A. (1989). A control systems framework for understanding normal and abnormal posture. American Journal of Occupational Therapy, 43(5), 291-301. doi: 10.5014/ajot.43.5.291

Mansur, C., Fregni, F., Boggio, P., Riberto, M., Gallucci-Neto, J., Santos, C., et al. (2005). A Sham stimulation-controlled trial of 
rTMS of the unaffected hemisphere in stroke patients. Neurology, 64(10), 1802-04. doi: 10.1212/01.wnl.0000161839.38079.92

Mathers, C., Fat, D. M., \& Boerma, J. T. (2008). The global burden of disease. Geneva: World Health Organization.

Niimi, M., Hashimoto, K., Kakuda, W., Miyano, S., Momosaki, R., Ishima, T., et al. (2016). Role of brain-derived neurotrophic factor in beneficial effects of repetitive transcranial magnetic stimulation for upper limb hemipare-sis after stroke. PLOS ONE, 11(3), e0152241. doi: 10.1371/journal.pone.0152241

Paternostro-Sluga, T., Grim-Stieger, M., Posch, M., Schuhfried, O., Vacariu, G., Mittermaier, C., et al. (2008). Reliability and validity of the Medical Research Council (MRC) scale and a modified scale for testing muscle strength in patients with radial palsy. Journal of Rehabilitation Medicine, 40(8), 665-71. doi: 10.2340/16501977-0235

Rode, G., Tiliket, C., \& Boisson, D. (1998). Predominance of postural imbalance in left hemiparetic patients. Scandinavian Journal of Rehabilitation Medicine, 29(1), 11-16. PMID: 9084100

Rossini, P., Barker, A., Berardelli, A., Caramia, M., Caruso, G., Cracco, R., et al. (1994). Non-invasive electrical and magnetic stimulation of the brain, spinal cord and roots: basic principles and procedures for routine clinical application. Report of an IFCN committee. Electroencephalography and Clinical Neurophysiology, 91(2), 79-92. doi: 10.1016/0013-4694(94)90029-9

Rossini, P., Caltagirone, C., Castriota-Scanderbeg, A., Cicinelli, P., Del Gratta, C., Demartin, M., et al. (1998). Hand motor cortical area reorganization in stroke: A study with fMRI, MEG and TCS maps. Neuroreport, 9(9), 2141-46. doi 10.1097/00001756-199806220-00043

Shimizu, T., Hosaki, A., Hino, T., Sato, M., Komori, T., Hirai, S., et al. (2002). Motor cortical disinhibition in the unaffected hemisphere after unilateral cortical stroke. Brain, 125(8), 18961907. doi: $10.1093 /$ brain/awf183

Speer, A. M., Kimbrell, T. A., Wassermann, E. M., D Repella, J., Willis, M. W., Herscovitch, P., et al. (2000). Opposite effects of high and low frequency rTMS on regional brain activity in depressed patients. Biological Psychiatry, 48(12), 1133-41. doi: 10.1016/s0006-3223(00)01065-9

Steffen, T. M., Hacker, T. A., \& Mollinger, L. (2002). Age-and gender-related test performance in community-dwelling elderly people: Six-Minute Walk Test, Berg Balance Scale, Timed Up \& Go Test, and gait speeds. Physical Therapy, 82(2), 128-137. doi: $10.1093 / \mathrm{ptj} / 82.2 .128$

Takeuchi, N., Chuma, T., Matsuo, Y., Watanabe, I., \& Ikoma, K. (2005). Repetitive transcranial magnetic stimulation of contralesional primary motor cortex improves hand function after stroke. Stroke, 36(12), 2681-86. doi: 10.1161/01. str.0000189658.51972.34

Traversa, R., Cicinelli, P., Pasqualetti, P., Filippi, M., \& Rossini, P. M. (1998). Follow-up of interhemispheric differences of motor evoked potentials from theaffected'andunaffected'hemispher es in human stroke. Brain Research, 803(1-2), 1-8. doi: 10.1016/ s0006-8993(98)00505-8

Ward, N. S., \& Frackowiak, R. S. (2006). The functional anatomy of cerebral reorganisation after focal brain injury. Journal of Physiology-Paris, 99(4-6), 425-436. doi: 10.1016/j.jphysparis. 2006.03 .002
Wassermann, E. M., \& Lisanby, S. H. (2001). Therapeutic application of repetitive transcranial magnetic stimulation: A review. Clinical Neurophysiology, 112(8), 1367-77. doi: 10.1016/ s1388-2457(01)00585-5

Weiduschat, N., Thiel, A., Rubi-Fessen, I., Hartmann, A., Kessler, J., Merl, P., et al. (2011). Effects of repetitive transcranial magnetic stimulation in aphasic stroke a randomized controlled pilot study. Stroke, 42(2), 409-415. doi: 10.1161/strokeaha.110.597864 
